\title{
SINGLET FISSION IN ORGANIC CRYSTALS
}

\author{
LIN MA \\ Division of Physics and Applied Physics, School of Physical and Mathematical Sciences, \\ Nanyang Technological University, 637371, Singapore \\ CHRISTIAN KLOC \\ Division of Materials Science, School of Materials Science and Engineering, Nanyang \\ Technological University, 639798, Singapore \\ CESARE SOCI \\ Division of Physics and Applied Physics, School of Physical and Mathematical Sciences, \\ Nanyang Technological University, 637371, Singapore \\ MARIA E. MICHEL-BEYERLE \\ Division of Physics and Applied Physics, School of Physical and Mathematical Sciences, \\ Nanyang Technological University, 637371, Singapore \\ GAGIK G. GURZADYAN \\ Division of Physics and Applied Physics, School of Physical and Mathematical Sciences, \\ Nanyang Technological University, 637371, Singapore
}

\begin{abstract}
Singlet fission attracts an extensive attention in recent years due to its potential to improve the efficiency of organic solar cells. Ultrafast time-resolved measurements provide a powerful tool for direct determination of singlet fission pathways and rates. In this chapter we present and discuss the experimental data on one- and two-photon, as well as consecutive two-quantum induced singlet fission in organic crystals rubrene and perylene. Temperature and excitation wavelength dependent rates of the singlet fission are discussed in terms of the activation energy. Ultrafast singlet fission was demonstrated to proceed directly from upper vibrational states of $S_{1}$, from upper excited states $S_{N}$, bypassing relaxed $\mathrm{S}_{1}$ state, as well as from two-photon excited states.
\end{abstract}

\section{Introduction}

\subsection{Singlet fission}

Singlet fission is a process in which a singlet excited molecule, usually in a densely packed organic solid, shares its energy with a neighboring molecule in 
its electronic ground state, both molecules forming a pair of triplet states in a spin allowed process. ${ }^{1}$ Singlet fission was first proposed in 1965 to explain the delayed fluorescence in anthracene crystal. ${ }^{2}$ And it is proven by the magnetic field effect study on tetracene crystal by Geacintov ${ }^{3}$ and Merrifield ${ }^{4}$ in 1969. Last decade, with the boom and development in solar energy as the clean energy source, increase of the efficiency of the solar cells becomes a hot issue.

The thermodynamic limit of energy conversion efficiency in single cell photovoltaic devices is determined by the spectral mismatch between the solar radiation and the absorption of the active semiconductor. Two factors affect this limit, hence the maximum efficiency attainable in single-junction cells: 1. solar photons with energy lower than the optical bandgap of the semiconductor are not absorbed; 2 . photons with energy higher than the bandgap generally produce a single electron-hole pair, which means the energy in excess of the bandgap energy is lost via thermalization processes. Once the bandgap of the active semiconductor has been optimized to match the solar spectrum, one way to increase the thermodynamic efficiency in single cell photovoltaics is the generation of multiple electron-hole pairs per absorbed photon with energy in excess of the bandgap. ${ }^{5,6}$ This process, which in bulk semiconductors is known as impact-ionization and in low-dimensional systems is often referred to as Multiple-Exciton Generation (MEG), ${ }^{7,8}$ can be highly enhanced in quantum dots thanks to the large electron-electron interaction resulting from quantum confinement. MEG was also reported in the case of semiconducting singlewalled carbon nanotubes (SWNTs), where absorption of single photons with energies corresponding to three times the energy gap of $(6,5)$ SWNTs resulted in an exciton generation efficiency of $130 \%$ per single photon absorbed. ${ }^{9}$

Singlet fission is an analogue of the MEG in the organic materials; the difference is that in this case, triplet excitons are generated from singlet exciton with the theoretical quantum yield up to $200 \%$. The recent development and advances on singlet fission are reviewed in detail by Smith and Michl. ${ }^{10,11}$ Singlet fission in semiconducting polymer thienylenevinylene was recently reported in Ref. 12.

Here, we present results of our studies on singlet fission in organic crystals rubrene and perylene. Singlet fission from upper excited electronic states as well as singlet fission induced via two-photon or consecutive two-quantum (two-step) absorption are discussed in detail. 


\section{Materials and Methods}

Rubrene and perylene single crystals were grown by physical vapor transport (PVT) technique. ${ }^{13}$ The reddish source powder of rubrene (SigmaAldrich, $>99 \%$ purity) was purified by sublimation for at least 2 times before the crystal growth. The carrier gases used in PVT technique are Argon (Ar), Nitrogen $\left(\mathrm{N}_{2}\right)$, Hydrogen $\left(\mathrm{H}_{2}\right)$ and forming gas which is a mixture of $\mathrm{H}_{2}$ and $\mathrm{N}_{2}$ with varied mole fraction. Semi-transparent two-zone furnace is used to grow the crystal. Rubrene source powder is placed at the zone of $300^{\circ} \mathrm{C}$ and the other zone is set to a slightly lower temperature at $260^{\circ} \mathrm{C}$. The carrier gas flows from high temperature zone to the lower, thus the sublimated rubrene molecules are condensed at the wall of the inner quartz tube at the lower temperature zone. The harvested as-grown rubrene single crystals are mostly flakes or needles shape, the size can reach $10-15 \mathrm{~mm}$. They are selected under polarized microscope, only those who with smooth surface and large size are chosen for steady-state and transient absorption and fluorescence measurements. The thickness of rubrene single crystal used in the experiment is about $10 \mu \mathrm{m}$, characterized by SEM and steady state absorption spectrum.

Steady state absorption spectra were obtained by a UV-Vis spectrophotometer (Cary 100Bio, Varian) at $1.0 \mathrm{~nm}$ resolution, and the steady state fluorescence emission and excitation spectra were recorded by the spectrofluorometer (Fluorolog-3, HORIBA Jobin Yvon).

The transient absorption (TA) spectra were measured by the optical femtosecond pump probe spectroscopy; the output of titanium-sapphire (Legend Elite, Coherent) regenerative amplifier seeded by the oscillator (Micra, Coherent) was used as a pulse laser source. The fundamental wavelength was $800 \mathrm{~nm}$, pulse width $65 \mathrm{fs}$, pulse repetition rate $1 \mathrm{kHz}$, and average power 3.5 W. $90 \%$ of the fundamental beam was converted into the visible and UV range by use of optical parametric oscillator (Topas, Light Conversion) and secondand sum-frequency mixing nonlinear processes. The remaining $10 \%$ was used to generate white light continuum in $\mathrm{CaF}_{2}$ plate, i.e. probe beam. The details on the pump probe setup and measurement method are described in Ref. 14.

Time-resolved photoluminescence (TRPL) measurements were carried out at room temperature by time-correlated single photon counting (TCSPC) technique with resolution of 10 ps (PicoQuant PicoHarp 300). The output of Titanium sapphire laser (Chameleon, Coherent Inc. $100 \mathrm{fs}, 80 \mathrm{MHz}$ ) with second- and third harmonic generation was used for exciting the fluorescence. The temperature dependent time-resolved fluorescence was measured by combining the TCSPC technique with an exchange gas continuous flow cryostat 


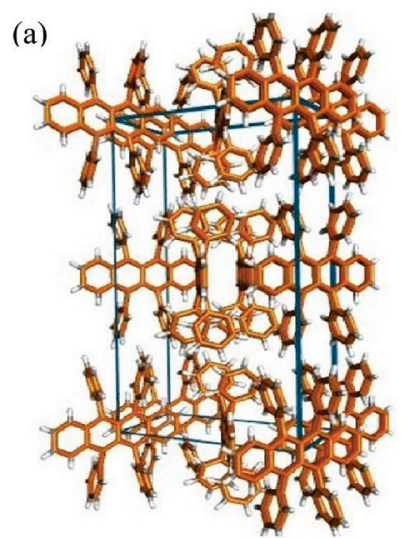

(b)

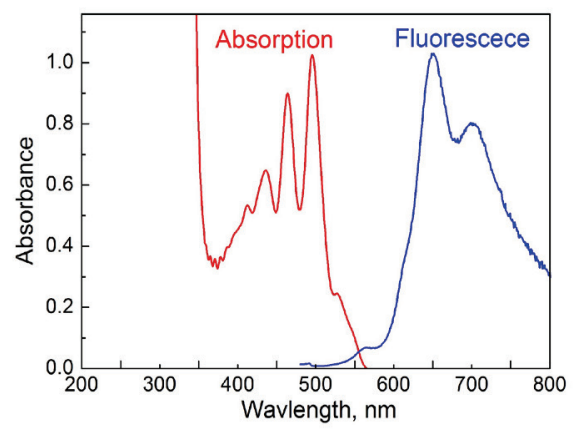

Figure 1. Rubrene crystal structure ${ }^{15}$ (a) and steady state absorption and fluorescence spectra ${ }^{16}$ (b).

(OptistatCF, Oxford Instruments).

The fluorescence up-conversion measurements were carried out by a commercial system FOG 100 (CDP Systems Corp., Russia). The second harmonic of a Titanium sapphire laser (Chameleon, Coherent Inc.) at $400 \mathrm{~nm}$ $(100 \mathrm{fs}, 80 \mathrm{MHz})$ was used as the excitation source. The fluorescence (540-700 $\mathrm{nm})$ was collected by parabolic mirror and focused into a $0.5 \mathrm{~mm}$ BBO crystal (cut angle $38^{\circ}$, ooe interaction) together with the fundamental radiation $(800 \mathrm{~nm}$ ) to generate the sum-frequency radiation. The resulting radiation $(322-373 \mathrm{~nm})$ after passing through a double monochromator (CDP2022D) was detected by a photomultiplier based photon counting electronics.

\section{Singlet Fission in Organic Crystals}

Singlet fission in rubrene and perylene single crystals was studied under various excitation conditions, i.e. one- and two-photon excitation, consecutive twoquantum excitation. Singlet fission from upper excited electronic states bypassing the lowest excited $\mathrm{S}_{1}$ state is reported and discussed in detail. Fission rates were found to depend on the excitation wavelength and temperature.

\subsection{Singlet fission: one quantum excitation}

\subsubsection{Singlet fission from the lowest excited singlet state $S_{1}$}

The rubrene crystal structure and the steady state spectra are presented in Fig. 1 (a) and (b). Transient absorption spectra in rubrene single crystal thin film were measured under $500 \mathrm{~nm}$ excitation (Fig. 2a). There are two positive bands with 

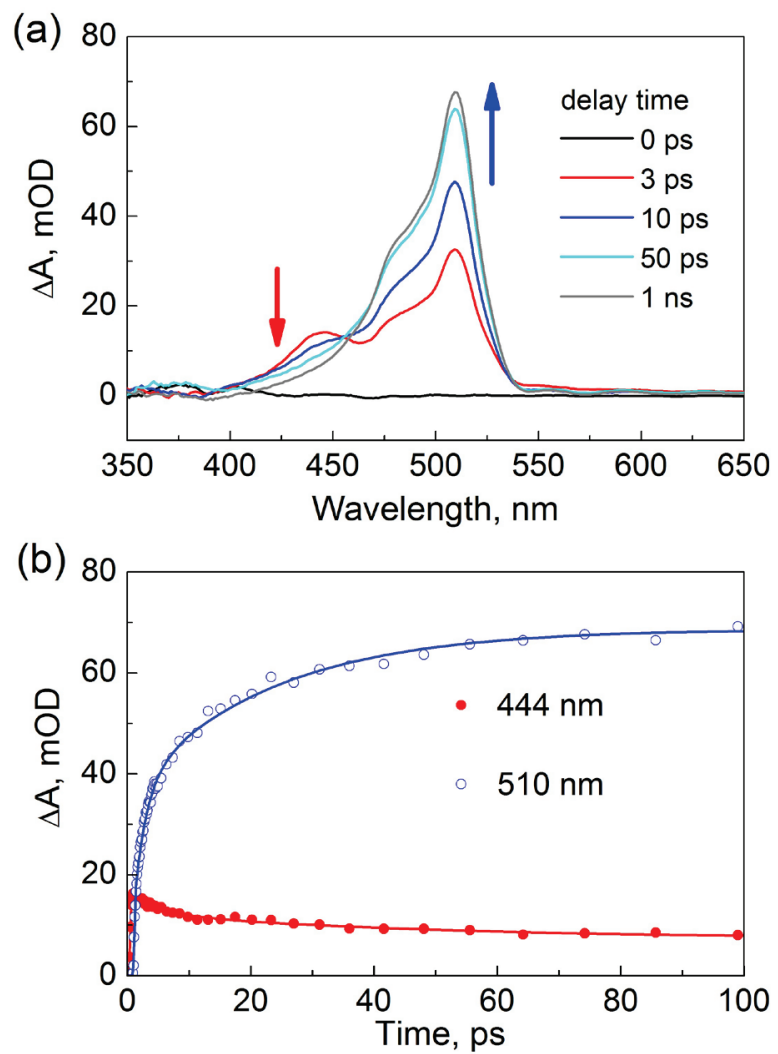

Figure 2. (a) Transient absorption spectra for the rubrene thin single crystal under $500 \mathrm{~nm}$ excitation. (b) Transient kinetics at 444 and $510 \mathrm{~nm}^{.16}$

maxima at 444 and $510 \mathrm{~nm}$. Transient band at $444 \mathrm{~nm}$ decays biexponentially with $\tau_{1}=5.2 \mathrm{ps}, \tau_{2}=51 \mathrm{ps}$ (Fig. $2 \mathrm{~b}$ ), which correlated well with the fluorescence lifetime TCSPC measurements: $40 \mathrm{ps}^{16}$. In consideration of the transient absorption spectra of the rubrene in solution, the $444 \mathrm{~nm}$ band of single crystal is assigned to the singlet-singlet absorption $\left(\mathrm{S}_{1} \rightarrow \mathrm{S}_{\mathrm{N}}\right){ }^{16}$

Transient band at $510 \mathrm{~nm}$ develops with two rise components $\tau_{1}=2.3 \mathrm{ps}, \tau_{2}$ $=23 \mathrm{ps}$ and lives long: doesn't show decay in $1 \mathrm{~ns}$ time window. For the $510 \mathrm{~nm}$ band, compared with the reported transient absorption results of the rubrene solution studied by flash-photolysis ${ }^{17}, 510 \mathrm{~nm}$ band position is in agreement with the triplet-triplet absorption $\mathrm{T}_{1} \rightarrow \mathrm{T}_{\mathrm{N}}(10 \mathrm{~nm}$ red shift). The decay of singlet state is accompanied by the formation of triplet states. An isosbestic point at $455 \mathrm{~nm}$ is an indication of a single transformation process, in which singlet states are 


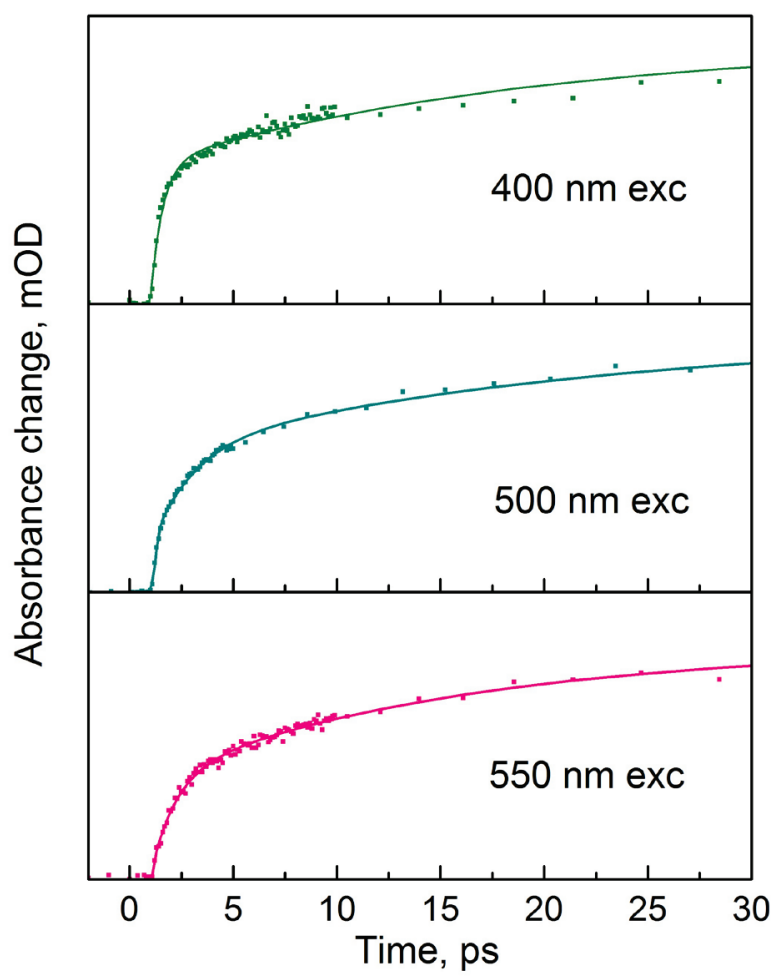

Figure 3. Transient absorption kinetics at $510 \mathrm{~nm}\left(\mathrm{~T}_{1}\right.$ state) under one-photon excitation (400$550 \mathrm{~nm})$.

converted into triplet states $\left(\mathrm{S}_{1} \rightarrow \mathrm{T}_{1}\right)$. Transient absorption spectrum was also measured for rubrene in toluene solution, however, only the TA band of $\mathrm{S}_{1}$ state was observed. Absence of triplet signal in solution is due to the low intersystem crossing efficiency $(0.05){ }^{17}$ Therefore, the triplet formation in crystal is attributed to the singlet fission process $\left(\mathrm{S}_{1} \rightarrow 2 \mathrm{~T}_{1}\right)$. The triplet state forms with two time constants $\tau_{1}=2.3 \mathrm{ps}$ and $\tau_{2}=23 \mathrm{ps}$, which corresponds to the direct fission from the upper vibrational states of $S_{1}$ and thermally activated fission from the lowest excited state $S_{1}$, respectively.

\subsubsection{Singlet fission from upper vibrational states of $S_{1}$}

We have applied various excitation wavelengths, 400, 500 and $550 \mathrm{~nm}$, in order to excite different vibrational states of the first excited singlet state $S_{1}$ in rubrene crystal. Comparison of the triplets' rise time under different excitation wavelengths is shown in Fig. 3, which indicates the rise time of triplet states, i.e., 

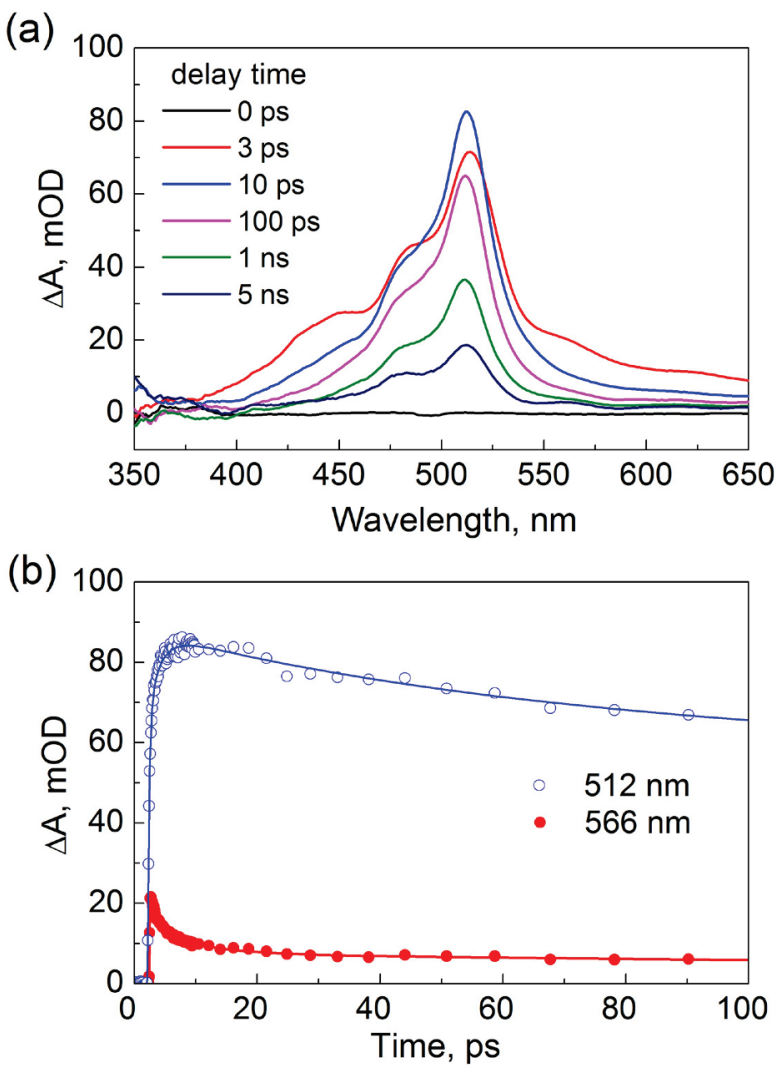

Figure 4. (a) Transient absorption spectra for the rubrene thin single crystal under $250 \mathrm{~nm}$ excitation. (b) Transient kinetics at 512 and $566 \mathrm{~nm}^{16}$

singlet fission rate, is pump wavelength dependent. ${ }^{18}$ The triplet states form faster with decreasing pump wavelength (i.e., increasing pump photon energy). It points out that singlet fission can proceed directly from the upper vibrational states of $S_{1}(400-550 \mathrm{~nm})$. Singlet fission is faster from the upper excited states since it is exoergic. Detailed explanation of this effect is described below (section 3.14 and 3.2).

\subsubsection{Singlet fission from upper excited singlet states $S_{N}$}

Transient absorption under $250 \mathrm{~nm}$ excitation is shown in Fig. 4. The main feature still comes from the two absorption bands of $\mathrm{S}_{1}$ and $\mathrm{T}_{1}$. Compared with the spectra under $500 \mathrm{~nm}$ excitation, the TA spectra become much broader. We assign this broadening to the formation of polaron. ${ }^{16}$ The isosbestic point at 


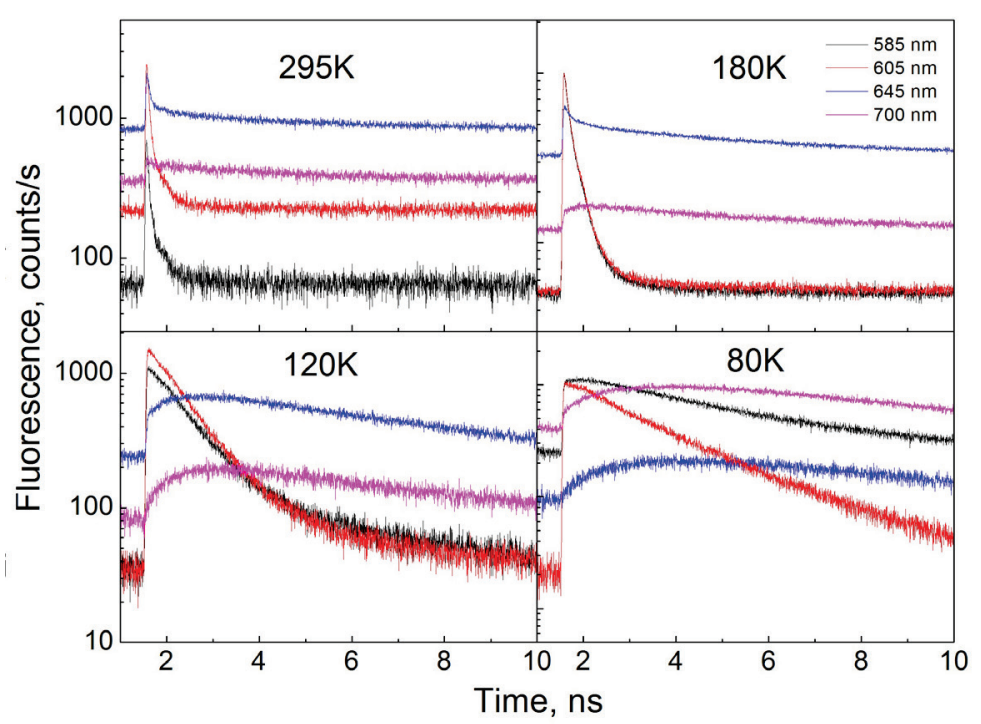

Figure 5. Temperature dependent TCSPC fluorescence kinetics in rubrene under $400 \mathrm{~nm}$ excitation. $^{19}$

$455 \mathrm{~nm}$ observed in the case of $500 \mathrm{~nm}$ excitation here has disappeared, which is indicative that there is more than one transformation processes. Moreover, the formation of triplet state becomes 10 times faster: $\tau_{1}=200 \mathrm{fs}$ and $\tau_{2}=2 \mathrm{ps}$. The $200 \mathrm{fs}$ ultrafast rise is due to direct fission from the upper excited singlet states $S_{N}$, i.e. $S_{N} \rightarrow 2 T_{1}$. It also indicates that the internal conversion from $S_{N}$ to $S_{1}$ is relatively slow: $\tau_{\mathrm{ic}}>200 \mathrm{fs} .{ }^{20}$ Singlet fission which was observed to occur directly from upper excited singlet states $\mathrm{S}_{\mathrm{N}}$ can be considered as violation of Vavilov law, which states that the quantum yield of fluorescence is independent of the excitation wavelength i.e. all photophysical processes necessarily proceed from the lowest excited states $S_{1}$ or $T_{1}$. Singlet fission from upper excited electronic state $1 \mathrm{~B}_{\mathrm{u}}$ bypassing lower state $2 \mathrm{~A}_{\mathrm{g}}$ was recently reported in the semiconducting polymer thienylenevinylene. ${ }^{12}$

\subsubsection{Singlet fission: temperature dependence}

Apart from the transient absorption spectroscopy, time resolved fluorescence also provides a direct way for studying the singlet fission. Since the fluorescence is quenched mainly due to the singlet fission, the fluorescence lifetime reflects the rate of singlet fission. The temperature dependent time-resolved fluorescence was studied in rubrene crystal in order to characterize the thermally activated singlet fission. Time-resolved single photon counting (TCSPC) technique was 


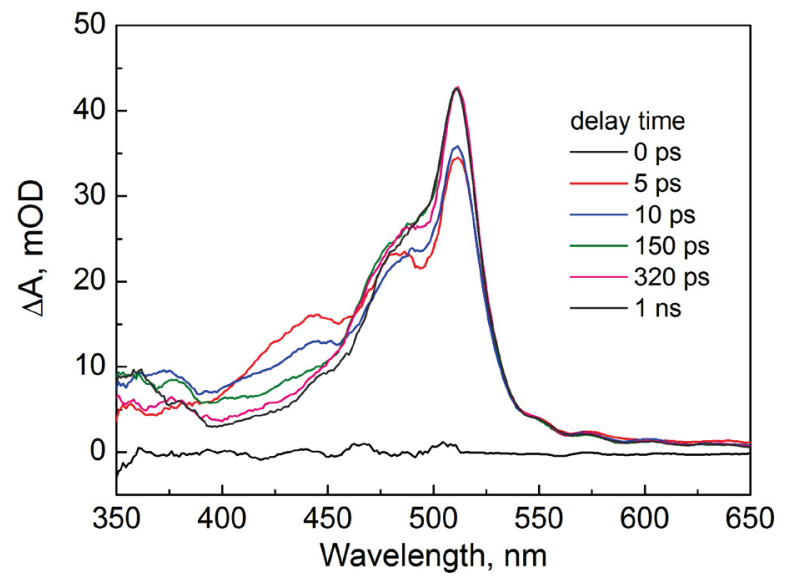

Figure 6. Transient absorption spectra under two-photon excitation at $750 \mathrm{~nm}$.

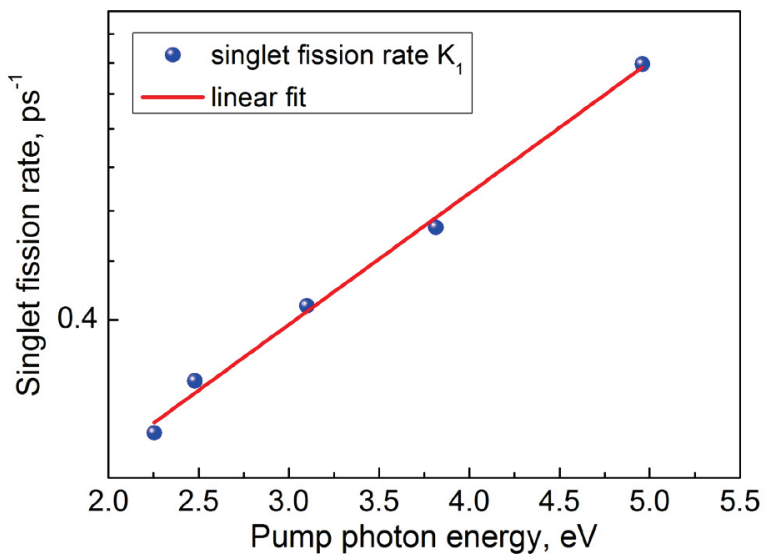

Figure 7. Arrhenius plot of singlet fission rate versus pump photon energy. ${ }^{18}$

applied for this purpose. Fluorescence kinetics at different emission wavelengths under various temperatures are shown in Fig. 5. At room temperature, all fluorescence kinetics are well fitted by two exponential components $\tau_{1}<100 \mathrm{ps}$ and $\tau_{2} \sim 3$ ns. For the blue wing of the fluorescence emission, i.e. 585 and 605 $\mathrm{nm}$, the short component $\tau_{1}$ becomes longer with decreasing temperature due to the suppression of singlet fission. Analyzing the temperature dependent fluorescence lifetimes, we found that the singlet fission rate follows Arrhenius law: 


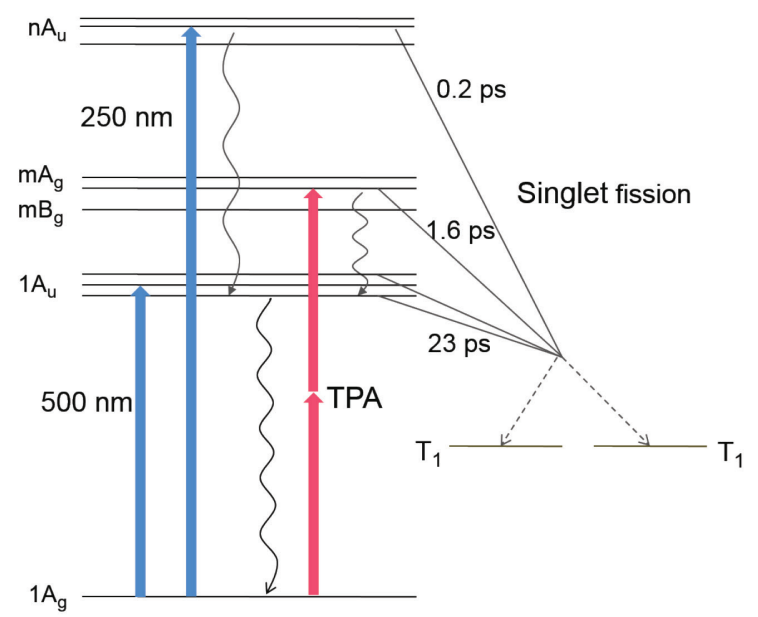

Figure 8. Schematic of the singlet fission induced by one- and two-photon absorption (TPA, 750 $\mathrm{nm}$ ) in rubrene crystal.

$$
k_{\text {fiss }}=A \exp \left(-\frac{E_{a}}{R T}\right)
$$

where $\mathrm{A}$ is the pre-exponential factor, $\mathrm{E}_{\mathrm{a}}$ is the activation energy, and $\mathrm{R}$ is the molar gas constant. The obtained activation energy $E_{a}$ is $0.05 \mathrm{eV}{ }^{19}$ which corresponds to the energy difference $\mathrm{E}\left(\mathrm{S}_{1}\right)-2 \mathrm{E}\left(\mathrm{T}_{1}\right)=-0.05 \mathrm{eV}{ }^{16}$

\subsection{Singlet fission: two-photon excitation}

In order to study the two-photon excited state relaxation processes in rubrene single crystal, we applied the femtosecond pump probe technique with $\lambda_{\text {exc }}=750$ $\mathrm{nm}$, i.e. no linear absorption. Although the two-photon excited states have different symmetries relative to the one-photon excited states, the singlet fission is still observed (Fig. 6). Transient band with the maximum at $510 \mathrm{~nm}$ corresponds to triplet-triplet absorption. The relation between the singlet fission rate $\mathrm{k}_{\text {fiss }}$ versus the pump photon energy is shown in Fig. 7: the $\ln \left(\mathrm{k}_{\text {fiss }}\right)$ undergoes a linear increase with excitation photon energy, i.e. follows the Arrhenius law (Eq. 1).

\subsection{Singlet fission in rubrene: conclusive remarks}

Based on the above, we have demonstrated that singlet fission can proceed directly from the upper vibrational levels of $S_{1}$, upper excited singlet states $S_{N}$, 


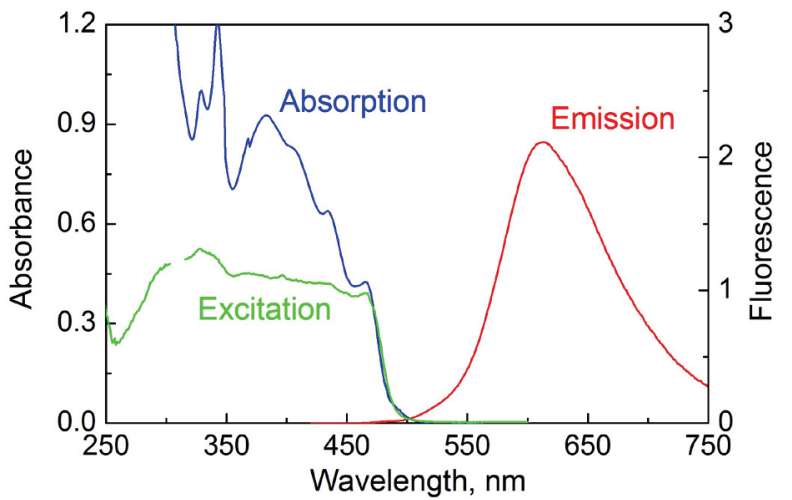

Figure 9. Steady state absorption, fluorescence and excitation spectra of $\alpha$-perylene crystal. ${ }^{21}$ For fluorescence emission spectrum $\lambda_{\mathrm{exc}}=400 \mathrm{~nm}$; for fluorescence excitation spectrum $\lambda_{\mathrm{em}}=620 \mathrm{~nm}$.

and two-photon excited states bypassing the relaxed $\mathrm{S}_{1}$ state. The relaxation process of rubrene single crystal under various excitation conditions is shown in Fig. 8. Singlet fission from the $S_{1}$ state proceeds within 2 and 20 ps due to direct fission from upper and lowest vibrational levels of $S_{1}$, respectively. From the $S_{N}$ states an ultrafast singlet fission occurs within 200 fs. Direct singlet fission from two-photon excited states takes place within $1.6 \mathrm{ps}$, and it competes with the fast internal conversion. $40 \%$ of the two-photon excited states relax to the lowest one-photon excited state $S_{1}$, where triplet states continue to be formed, even though 15 times slower via thermally activated fission.

\subsection{Singlet fission: consecutive two-quantum (two-step) excitation}

Singlet fission can proceed also after excitation of the high-lying excited electronic states via consecutive singlet-singlet absorption: $\mathrm{S}_{0} \rightarrow \mathrm{S}_{1} \rightarrow \mathrm{S}_{\mathrm{N}}$. In this case much lower radiation intensities are required compared with the above described two-photon excitation. This was demonstrated for the organic single crystal $\alpha$-perylene. ${ }^{21}$ Perylene and its derivatives are widely used in organic field effect transistors ${ }^{22}$, light emitting diodes ${ }^{23}$ and photovoltaics ${ }^{24}$. Perylene belongs to monoclinic crystal system and exists in two forms: $\alpha$ and $\beta .{ }^{25} \beta$-perylene is monomeric, with two molecules per unit cell; $\alpha$-perylene is dimeric, with four molecules in a unit cell.

\subsubsection{Steady-state absorption and fluorescence of perylene}

Steady state absorption, fluorescence emission and excitation spectra of $\alpha$ perylene are shown in Fig. 9. Due to the high absorption cross-section all optical 


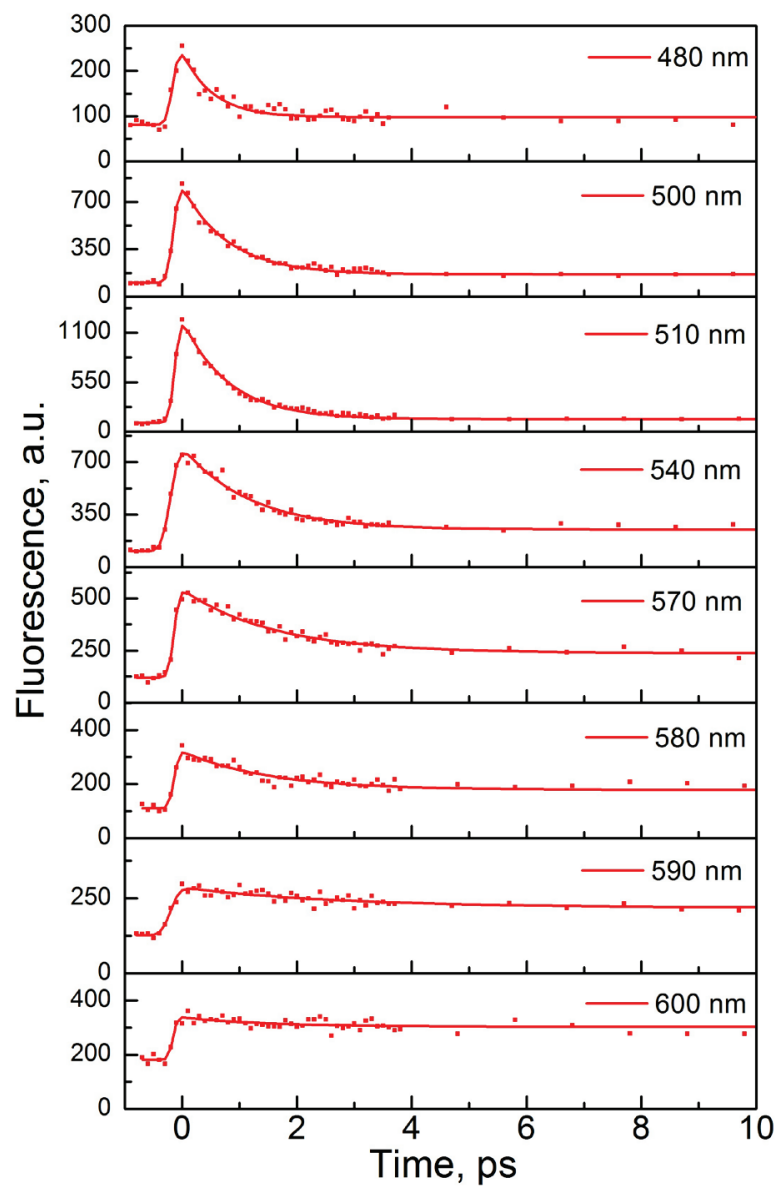

Figure 10. The fluorescence kinetics in $\alpha$-perylene crystal, measured by fluorescence upconversion under $400 \mathrm{~nm}$ excitation at different emission wavelengths.

measurements were performed with thin samples. The fluorescence centered at $615 \mathrm{~nm}$ corresponds to the well-known excimer (self-trapped exciton) emission in $\alpha$-perylene crystal. ${ }^{26}$ The fluorescence spectrum is invariant under different excitation wavelengths. A discrepancy between the absorption and fluorescence excitation spectra was found. It indicates that below $465 \mathrm{~nm}$ quenching takes place. Comparison of the absorption and fluorescence excitation spectra shows that they correspond well only above $465 \mathrm{~nm}$. However, below $465 \mathrm{~nm}$ there is a drop of the excitation spectrum indicating an efficient quenching process. This is due to dimer cation formation from ion pair (IP): the threshold according to Ref. 27 is $2.65 \mathrm{eV}$. At wavelengths below $350 \mathrm{~nm}$ there is a drastic drop of 

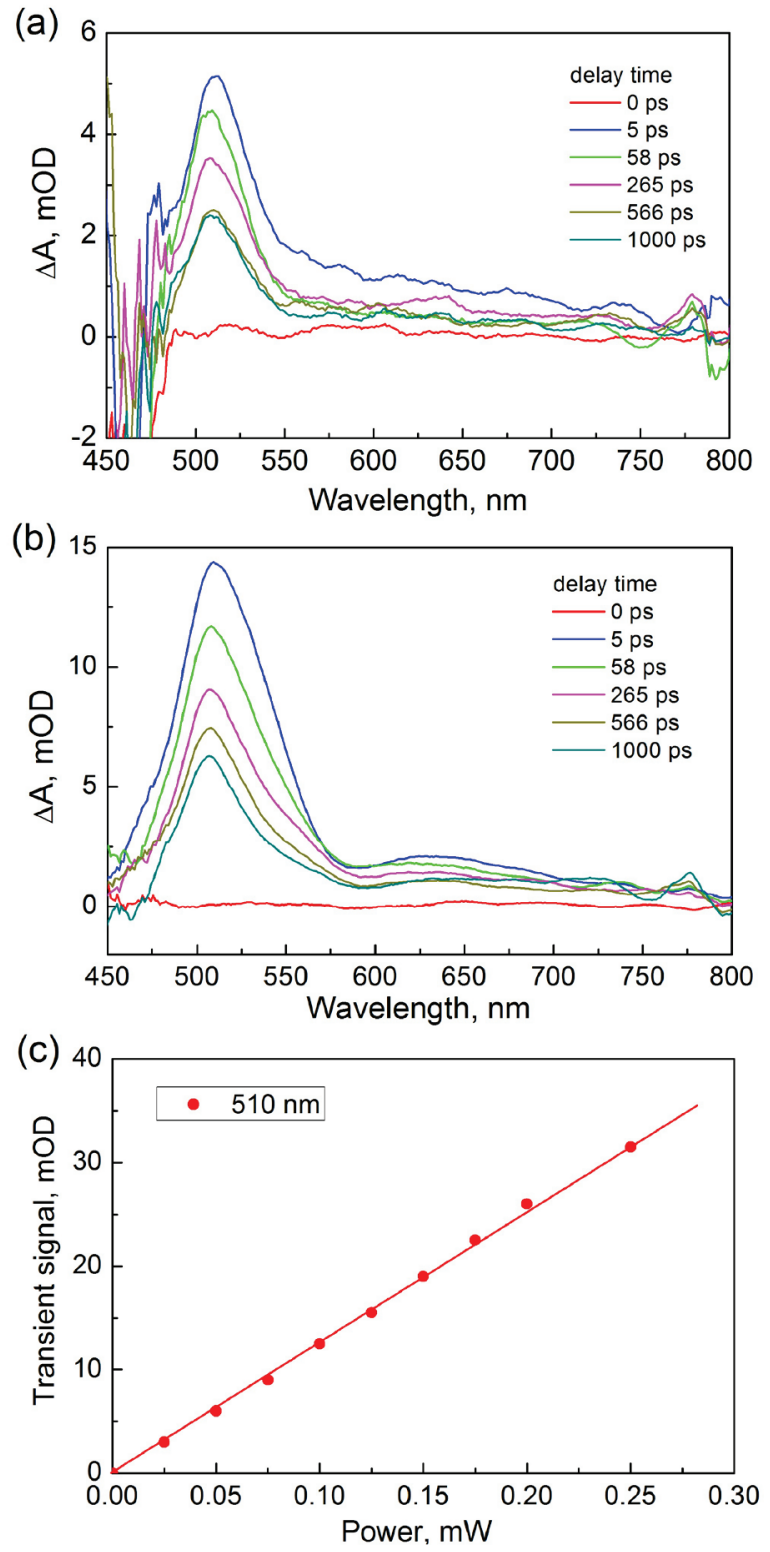

Figure 11. Transient absorption spectra for $\alpha$-perylene crystal thin film at different delay times; $\lambda_{\text {exc }}=250 \mathrm{~nm}$ (a) and $\lambda_{\text {exc }}=340 \mathrm{~nm}$ (b). The power dependence of $510 \mathrm{~nm}$ TA signal measured at $\lambda_{\mathrm{exc}}=340 \mathrm{~nm}(\mathrm{c}) .^{21}$ 
the fluorescence although the absorption becomes much larger. It is indicative of an efficient singlet fission which proceeds directly from the upper vibrational levels of $\mathrm{S}_{1}$. The threshold of this process is $3.51 \mathrm{eV}^{28,29}$

\subsubsection{Fluorescence decay}

The fluorescence up-conversion is applied in order to study the ultrafast dynamics of $\alpha$-perylene. ${ }^{21}$ The results are shown in Fig. 10. The fluorescence lifetime becomes longer with increasing emission wavelength. At $460 \mathrm{~nm}, 92 \%$ of the fluorescence decays with a time constant $\tau_{1}=500 \mathrm{fs}$. While at $630 \mathrm{~nm}$, fluorescence decays mainly with $\tau_{2}>1 \mathrm{~ns}$. The wavelength dependent fluorescence lifetime indicates that the $\mathrm{Y}$ state has a fine structure and includes many sublevels, with lifetimes ranging from 500 fs to 3 ps. We should note that no rise-time was detected for all the fluorescence kinetics due to the strong spectral overlap between different emitting states. Based on our time-resolved fluorescence measurements, we conclude that the excited monomeric state relaxes to the "hot" excimer state (Y state) within 100 fs. Then these "hot" excimers, i.e. different sublevels of $Y$ state, continue relaxing to the fully relaxed excimer state (E state) within 3 ps.

\subsubsection{Pump-probe measurements}

\subsubsection{One-quantum excitation: $\lambda=250$ and $340 \mathrm{~nm}$}

The TA spectra under $250 \mathrm{~nm}$ pump are shown in Fig. 11a. After the baseline correction (subtract the signal at negative delay times), the dominant TA peak locates at $505 \mathrm{~nm}$, accompanied by a broad absorption band ranging from 600 to $800 \mathrm{~nm}$. Similar spectra were also obtained at $\lambda_{\text {exc }}=340 \mathrm{~nm}$ (Fig. 11b). ${ }^{21}$ The power dependence of the transient signal at $510 \mathrm{~nm}$ is linear (Fig. 11c). Considering the excimer absorption in $\alpha$-perylene locating at $620 \mathrm{~nm},{ }^{30}$ the broad TA band is assigned to the excimer absorption, i.e. including $\mathrm{Y}$ and $\mathrm{E}$ states. Based on the discussion on the fluorescence upconversion measurements, the "hot" excimer state $\mathrm{Y}$ consists of various sublevels covering the range of $4500 \mathrm{~cm}^{-1}$. Therefore, the excimer TA band is broad. The $505 \mathrm{~nm}$ TA band is due to dimer cation; in perylene solution it is located at $503 \mathrm{~nm} .{ }^{31}$ In $\alpha$-perylene crystal, the ion pair (IP) state, the precursor for the charge carrier, locates at 2.65 $\mathrm{eV}(468 \mathrm{~nm}) .{ }^{27}$ It should be mentioned that at the negative delay times a pronounced peak located at $480 \mathrm{~nm}$ was observed, ${ }^{21}$ which corresponds to the triplet state absorption. In diluted solution, only excited singlet state absorption at $700 \mathrm{~nm}$ was observed. No triplet state was found due to the low intersystem crossing $\left(\phi_{\text {isc }}=0.01\right) .{ }^{32}$ Only in the highly concentrated solution we observed a 

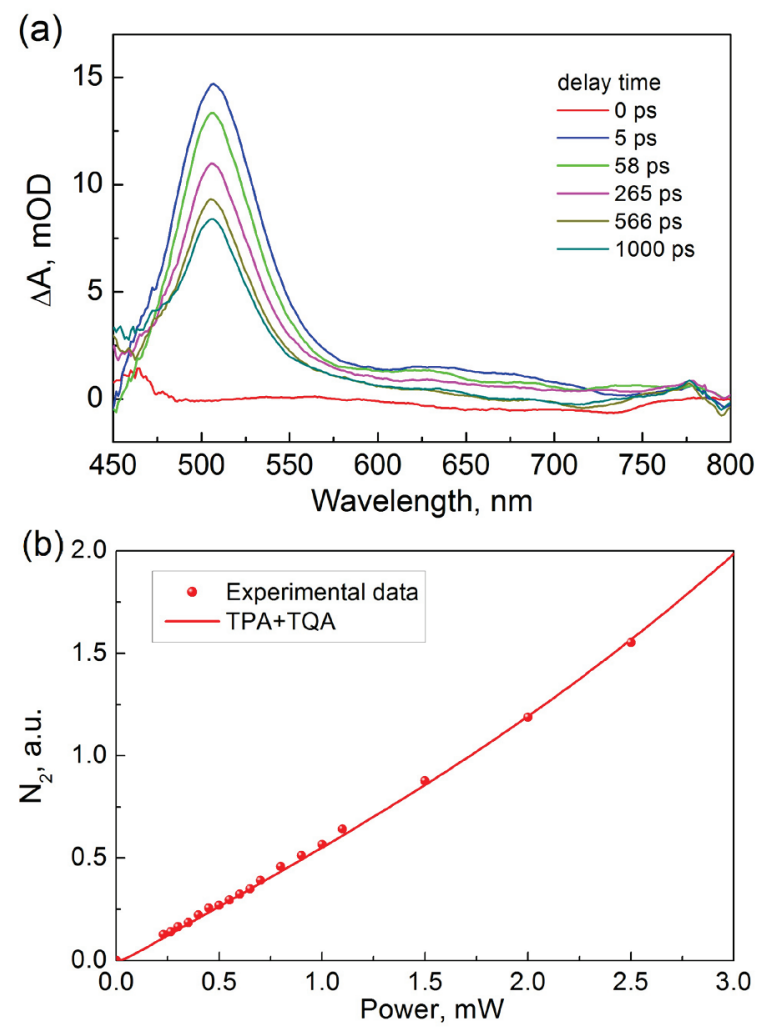

Figure 12. (a) Transient absorption spectra for $\alpha$-perylene crystal thin film at different delay times; $\lambda_{\mathrm{exc}}=475 \mathrm{~nm}$, (b) the power dependence of $510 \mathrm{~nm}$ TA signal. ${ }^{21}$

weak positive TA band at about $490 \mathrm{~nm}$ at $5 \mathrm{~ns}$ delay which corresponds to the triplet absorption. Therefore, the triplet states observed in $\alpha$-perylene crystal (Figs. 11a, b) are not generated by intersystem crossing. Hence triplet formation in the crystal is due to singlet fission. The only information in literature on singlet fission in $\alpha$-perylene crystal is related to the magnetic field effect under the excitation with xenon lamp. ${ }^{28,29}$ The threshold for singlet fission was estimated to be $3.51 \mathrm{eV}(353 \mathrm{~nm})$. The competing reaction was considered to be the fast excimer formation.

\subsubsection{Two-quantum excitation: $\lambda=475 \mathrm{~nm}$}

Figure 12a shows the TA spectra under excitation at $475 \mathrm{~nm}$, i.e. below the threshold for both fission and cation formation. ${ }^{21}$ These TA spectra are similar to that for 250 or $340 \mathrm{~nm}$ excitation. The TA signals from dimer cation and 
excimer still govern the whole spectra. Moreover, at the negative delay times, the triplet absorption is still the dominant. However, as mentioned above, neither cation generation nor singlet fission under one-photon absorption at $475 \mathrm{~nm}$ should be expected. The power dependence of the transient signal at $510 \mathrm{~nm}$ is nonlinear (Fig 12b). This power dependence is explained in terms of two-photon absorption (TPA, i.e., simultaneous absorption of two photons) and consecutive two-quantum absorption (TQA, i.e., two-step absorption of two photons via intermediate excimer state). ${ }^{21}$ Contribution of both TPA and TQA processes was discussed in detail in Refs. 33,34 for the nonlinear crystal KDP.

The rate equations for TQA process are: ${ }^{21,33-35}$

$$
\begin{gathered}
\frac{d N_{0}}{d t}=-\sigma_{1} I N_{0}+\frac{N_{1}}{\tau} \\
\frac{d N_{1}}{d t}=\sigma_{1} I N_{0}-\phi_{2} \sigma_{2} I N_{1}-\frac{N_{1}}{\tau} \\
\frac{d N(T Q A)}{d t}=\phi_{2} \sigma_{2} I N_{1}
\end{gathered}
$$

Where $\mathrm{N}_{0}, \mathrm{~N}_{1}$ and $\mathrm{N}(\mathrm{TQA})$ are the populations of the ground state $\left(\mathrm{S}_{0}\right)$, intermediate state $\left(\mathrm{S}_{1}\right)$ and the two quantum excited state $\left(\mathrm{S}_{\mathrm{N}}\right) . \sigma_{1}$ and $\sigma_{2}$ are the absorption cross sections of $\mathrm{S}_{0} \rightarrow \mathrm{S}_{1}$ and $\mathrm{S}_{1} \rightarrow \mathrm{S}_{\mathrm{N}}$ transitions, respectively $\left(\sigma_{1}=\right.$ $7.6 * 10^{-17} \mathrm{~cm}^{2}$ at $475 \mathrm{~nm}^{36}$ ). $\phi_{2}$ is the yield of the final product from the two quantum excited state $\mathrm{S}_{\mathrm{N}}$. Assuming rectangular pulse shape and based on different intermediate state lifetimes and the ratio of $\phi_{2} \sigma_{2}$ and $\sigma_{1}$, we have obtained the numerical solution of the rate equations (2)-(4). Taking also into consideration TPA process, a term N(TPA) $=\mathrm{A}^{*} \mathrm{I}^{2}$ is added to N(TQA). The best fit is obtained for $\phi_{2} \sigma_{2}=50 \sigma_{1}$ (Fig. 12b). The lifetime of $S_{1}$ is taken $t=2$ ps which corresponds to the "hot" excimer Y state. Based on above considerations, we suggest that both singlet fission and dimer cation formation under $475 \mathrm{~nm}$ are due to the coexistence of TPA and TQA.

\section{Acknowledgments}

We thank Keke Zhang, Ke Jie Tan and Hui Jiang for providing high quality rubrene and perylene crystals.

\section{References}

1. C. E. Swenberg and N. E. Geacintov, Organic Molecular Photophysics. (Wiley \& Sons Ltd., Chichester, Sussex, 1973), vol. 18. 
2. S. Singh, W. J. Jones, W. Siebrand, B. P. Stoicheff, and W. G. Schneider, J. Chem. Phys. 42, 330 (1965).

3. N. Geacintov, M. Pope, and F. Vogel, Phys. Rev. Lett. 22, 593 (1969).

4. R. E. Merrifield, P. Avakian, and R. P. Groff, Chem. Phys. Lett. 3, 386 (1969).

5. M. C. Hanna and A. J. Nozik, J. Appl. Phys. 100, 074510 (2006).

6. M. C. Beard and R. J. Ellingson, Laser \& Photon. Rev. 2, 377 (2008).

7. R. J. Cava, F. J. DiSalvo, L. E. Brus et al., Prog. Solid State Chem. 30, 1 (2002).

8. R. D. Schaller, J. M. Pietryga, and V. I. Klimov, Nano Lett. 7, 3469 (2007).

9. S. Wang, M. Khafizov, X. Tu, M. Zheng, and T. D. Krauss, Nano Lett. 10, 2381 (2010).

10. M. B. Smith and J. Michl, Chem. Rev. 110, 6891 (2010).

11. M. B. Smith and J. Michl, Annu. Rev. Phys. Chem. 64, 361 (2013).

12. A. J. Musser, M. Al-Hashimi, M. Maiuri, D. Brida, M. Heeney, G. Cerullo, R. H. Friend, and J. Clark, J. Am. Chem. Soc. 135, 12747 (2013).

13. C. Kloc, P. G. Simpkins, T. Siegrist, and R. A. Laudise, J. Cryst. Growth 182, 416 (1997).

14. J. Shang, T. Yu, J. Lin, and G. G. Gurzadyan, ACS Nano 5, 3278 (2011).

15. O. D. Jurchescu, A. Meetsma, and T. T. M. Palstra, Acta Crystallogr. Sect. $B$ 62, 330 (2006).

16. L. Ma, K. Zhang, C. Kloc, H.Sun, M. E. Michel-Beyerle, and G. G. Gurzadyan, Phys. Chem. Chem. Phys., 14, 8307 (2012).

17. A. Yildiz, P. T. Kissinger, and C. N. Reilley, J. Chem. Phys. 49, 1403 (1968).

18. L. Ma, G. Galstyan, K. Zhang, C. Kloc, H. Sun, C. Soci, M. E. MichelBeyerle, and G. G. Gurzadyan, J. Chem. Phys. 138, 184508 (2013).

19. L. Ma, K. Zhang, C. Kloc, H. Sun, C. Soci, M. E. Michel-Beyerle, and G. G. Gurzadyan, Phys. Rev. B 87, 201203 (2013).

20. W. G. Albrecht, H. Coufal, R. Haberkorn, and M. E. Michel-Beyerle, Phys. Stat. Sol. B 89, 261 (1978).

21. L. Ma, K. J. Tan, H. Jiang, C. Kloc, M. E. Michel-Beyerl, and G. G. Gurzadyan, (2013), in press.

22. B. A. Jones, M. J. Ahrens, M.-H. Yoon, A. Facchetti, T. J. Marks, and M. R. Wasielewski, Angew. Chem. Int. Ed. 43, 6363 (2004).

23. M. A. Angadi, D. Gosztola, and M. R. Wasielewski, Mater. Sci. Eng. B 63, 191 (1999).

24. L. Schmidt-Mende, A. Fechtenkötter, K. Müllen, E. Moons, R. H. Friend, and J. D. MacKenzie, Science 293, 1119 (2001).

25. J. Tanaka, Bull. Chem. Soc. Jpn. 36, 1237 (1963).

26. T. Y. H. Nishimura, K. Mizuno, M. Iemura and A. Matsui, J. Phys. Soc. Jpn. 53, 3999 (1984). 
27. A. Furube, M. Murai, Y. Tamaki, S. Watanabe, and R. Katoh, J. Phys. Chem. A 110, 6465 (2006).

28. W. G. Albrecht, M. E. Michel-Beyerle, and V. Yakhot, J. Lumin. 20, 147 (1979).

29. W. G. Albrecht, M. E. Michel-Beyerle, and V. Yakhot, Chem. Phys. 35, 193 (1978).

30. Z. Ludmer, L. Zeiri, and S. Starobinets, Phys. Rev. Lett. 48, 341 (1982).

31. K. Kimura, T. Yamazaki, and S. Katsumata, J. Phys. Chem. 75, 1768 (1971).

32. C. A. Parker and T. A. Joyce, Chem. Commun. (London) 0, 108b (1966).

33. G. G. Gurzadyan and R. K. Ispiryan, Appl. Phys. Lett. 59, 630 (1991).

34. G. G. Gurzadyan and R. K. Ispiryan, J. Nonlinear Optic. Phys. Mat. 01, 533 (1992).

35. Z. Shaquiri, E. Keskinova, A. Spassky, and D. Angelov, Photochem. Photobiol. 65, 517 (1997).

36. R. M. Hochstrasser, J. Chem. Phys. 40, 2559 (1964). 